\title{
Minimally Invasive Elective Surgery as a Treatment of Bowel Invagination in a Peutz-Jeghers Syndrome Case
}

\author{
Alberto García Picazo Beatriz López de la Torre Alfredo Vivas \\ Francisco J. García Eduardo Ferrero \\ General Surgery and Abdominal Organs Transplantation Department, 12th October \\ University Hospital, Complutense University of Madrid, Madrid, Spain
}

\section{Keywords}

Peutz-Jeghers syndrome $\cdot$ Abdominal pain $\cdot$ Small bowel obstruction $\cdot$ Case report

\begin{abstract}
We present a case of a 24-year-old woman with Peutz-Jeghers syndrome, recurrent colic abdominal pain, and lower gastrointestinal bleed for the last 5 years. Colonoscopy showed hamartomas without any dysplasia. In the enteral magnetic resonance imaging, a distal jejunum and ileum invagination, secondary to hamartomas was detected. The patient was referred to the Surgery Department and despite few symptoms, elective surgery was proposed. By laparoscopic surgery approach, the entire bowel was carefully revised, 3 intussusceptions and bowel volvulus were found, 2 in jejunum and 1 in ileum, causing incomplete obstruction and intestinal dilatation, with a diameter of $6 \mathrm{~cm}$. These intussusception areas were marked with a silk filament, after achieving devolvulation and disinvagination. A 5-cm laparotomy was done, to externalize the entire bowel, to explore it manually, to verify the absence of other lesions, and locate silk points. By longitudinal enterotomies on the antimesenteric intestinal border where silk filaments were located, the polyps were removed through their stalk, and the enterotomies were transversely closed. Postoperative evolution was favorable, starting oral tolerance on the fourth day and being discharged from the hospital on the seventh day. Eight months later, the patient was asymptomatic with a better quality of life.
\end{abstract}

(C) 2021 The Author(s).

Published by S. Karger AG, Basel

\section{Introduction}

Peutz-Jeghers syndrome (PJS) is an uncommon autosomal dominant genetic disease, included in the familiar syndromes of polyps, such as Cowden and juvenile polyposis [1]. It is a rare disease $(1 / 20,000)$, described first in 1921 (Peutz) and 1949 (Jeghers) [2, 3]. 
The majority of the cases are diagnosed between 12 and 30 years, appearing mostly in males [1]. Other possible tumors have been remarked to PJS, such as gynecological (ovary [21\%], uterus [9\%], breast [56\%], pancreas [36\%], and stomach [29\%]) [4].

The main symptoms are in the gastrointestinal tract, with multiple hamartomas in the small bowel and colon. Those lesions might generate, in long term, recurrent abdominal pain (23\%), lower gastrointestinal bleed (14\%), and intestinal obstruction (14\%) on account of intussusception (40\%) [5]. In some cases, elective or urgent surgery is indicated (10-20\%), mainly when these symptoms decrease quality of life, like in our case.

\section{Case Report/Case Presentation}

A 24-year-old woman had a history of high blood pressure, left ovarian cyst $(37 \times 27 \mathrm{~mm})$, and PJS, detected when she was 11 years old (mutation of IVS3-2A>G), and no family history was found. Medical monitoring was done by a gastroenterologist and the patient was referred to the Surgery Department because of intermittent abdominal pain related to intake and recurrent lower gastrointestinal bleed. Physical examination and laboratory tests did not reveal any abnormalities.

Radiological intestinal transit time did not present any alterations. Colonoscopy showed one polyp in the left-side colon (diameter of $3 \mathrm{~cm}$ ), one in the sigmoid colon (diameter of $2 \mathrm{~cm}$ ), and the last one in the rectum (diameter of $0.7 \mathrm{~cm}$ ), and polypectomy was done in all of them. In the pathology report, the first 2 polyps were hamartomas, and the last one a serrated polyp with lowgrade dysplasia (Fig. 1). On magnetic resonance imaging with enteral contrast, 2 jejunal-ileum intussusception areas (10-12 cm) were found, located in the hypogastric region, where existed a nodular indeterminate image $(3.5 \times 3.2 \mathrm{~cm})$ which produced proximal dilatation of the rest of the small bowel, with a maximum diameter of $2.8 \mathrm{~cm}$ and distal decompression. Elective surgical intervention was offered to remove all the polyps and revise the rest of the bowel.

By a laparoscopic surgery approach, the small bowel was completely explored. Generally, dilatation, with a maximum diameter of $6 \mathrm{~cm}$, and incomplete obstruction, secondary to bowel volvulus and 2 intussusceptions in jejunum, 40 and $80 \mathrm{~cm}$, respectively, from the Treitz angle $(2.5 \times 2$ and $3.5 \times 3.2 \mathrm{~cm})$ are observed; all were disinvaginated, devolvulated, and marked with a silk filament of 2/0 (Fig. 2). A 5-cm laparotomy was done to externalize the entire bowel and revised it manually; as a result, one ileum invagination was discovered (1.5 $\times 1 \mathrm{~cm}$ ) and marked with silk. Longitudinal enterotomies on the antimesenteric bowel borders were performed to the areas pointed with the silk (Fig. 3). This action allowed removal of those lesions and sectioning the stalk with an endovascular gastrointestinal anastomosis stapler, without doing bowel resections. The enterotomies were transversely closed with endovascular gastrointestinal anastomosis staplers and absorbable suture of 3/0 (Fig. 3). In the anatomopathological examination, all the polyps were hamartomas. Postoperative evolution was favorable with no complications. Oral tolerance was started on the fourth day, and hospital discharge was on the seventh day. In the last medical examination, 6 months after surgery, the patient was asymptomatic with a better quality of life.

\section{Discussion/Conclusion}

PJS is a dominant autosomal genetic disease, with a prevalence of $1 / 200,000$ [6]. In the majority of the cases, it is caused by a mutation of a kinase (STK11/LKB1) in 19p13.3 [7]. STK11 and LBB1 have been associated with the cell cycle and could avoid apoptosis by abnormal cells, generating multiple hamartomas [8,9].

\section{Karger'}




\section{Case Reports in Gastroenterology}
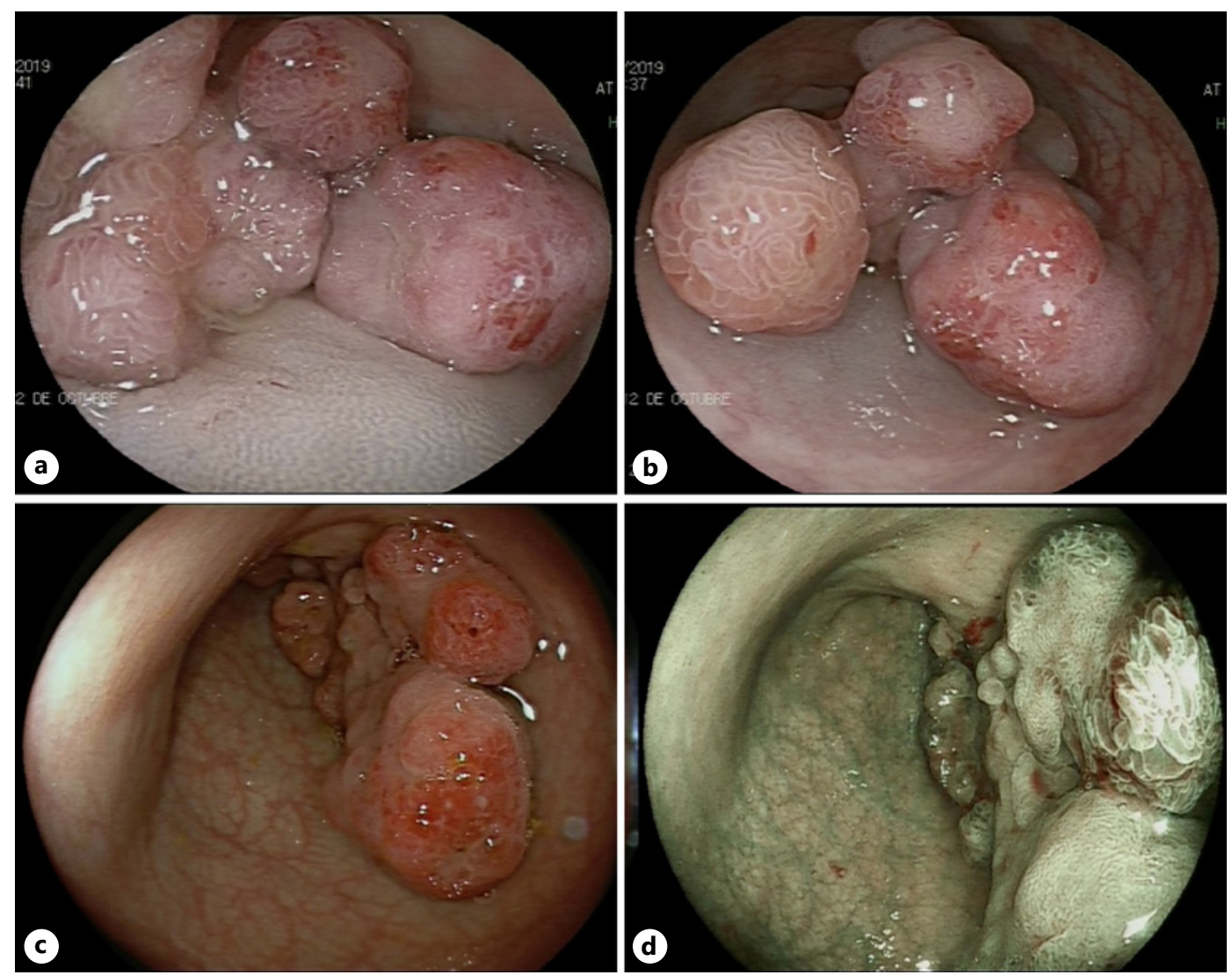

Fig. 1. Lower gastrointestinal endoscopy. Enormous polyps surrounding the entire colon surface (a, b), colonic lateral polyp (c), virtual chromoendoscopy (d) showing some areas with irregular glandular pattern.

The major proportion of polyps is in the small bowel and colon (65\%), stomach (50\%), and rectum (32\%), although they can also form in the extra-gastrointestinal system, such as the lungs, bladder, and renal pelvis. Histological analysis would reveal a typical hamartoma structure: smooth muscle proliferation near the muscular mucosa, without affecting the epithelium. The first life decade is associated with the growth period, whereas in the third, symptoms are generated, which might be recurrent abdominal pain (23\%), lower gastrointestinal bleed (14\%), and intestinal obstruction (14\%) as a result of intussusception (40\%).

Unfortunately, the cancer risk related to PJS is present; therefore, a strict follow-up has to be done in every patient. The malignancy risk is over $90 \%$ from the age of 60 years, with the most prevalent tumors occurring in the breast (54\%), colon (40\%), pancreas (36\%), stomach (30\%), ovaries (21\%), and small bowel (15\%). Other tumors are rarely described, for instance, Sertoli cell, lung, cervix, and desmoid tumors, where the main feature is rapid growth [10]. Meanwhile, gastrointestinal polyps rarely lead to malignancy, so prophylactic resection is not indicated.

Intestinal obstruction affects $20-40 \%$ of the patients, with the risk increasing with age; in the majority of the cases, it is secondary to small bowel intussusception by hamartomas. Endoscopy has poor effectiveness. Nowadays, new therapies are proposed such as small bowel polypectomy with balloon-assisted enteroscopy [11]. Surgical treatment includes disinvagination, trying to avoid intestinal resections, like in our patient. This aspect is essential because these patients could finally develop short bowel syndrome, as a result of recurrent episodes of intestinal obstruction [12]. In such cases, small bowel transplantation could be a therapeutic option [13]. 


\section{Case Reports in Gastroenterology}

García Picazo et al.: Minimally Invasive Surgery of Bowel Invagination in Peutz-Jeghers Syndrome
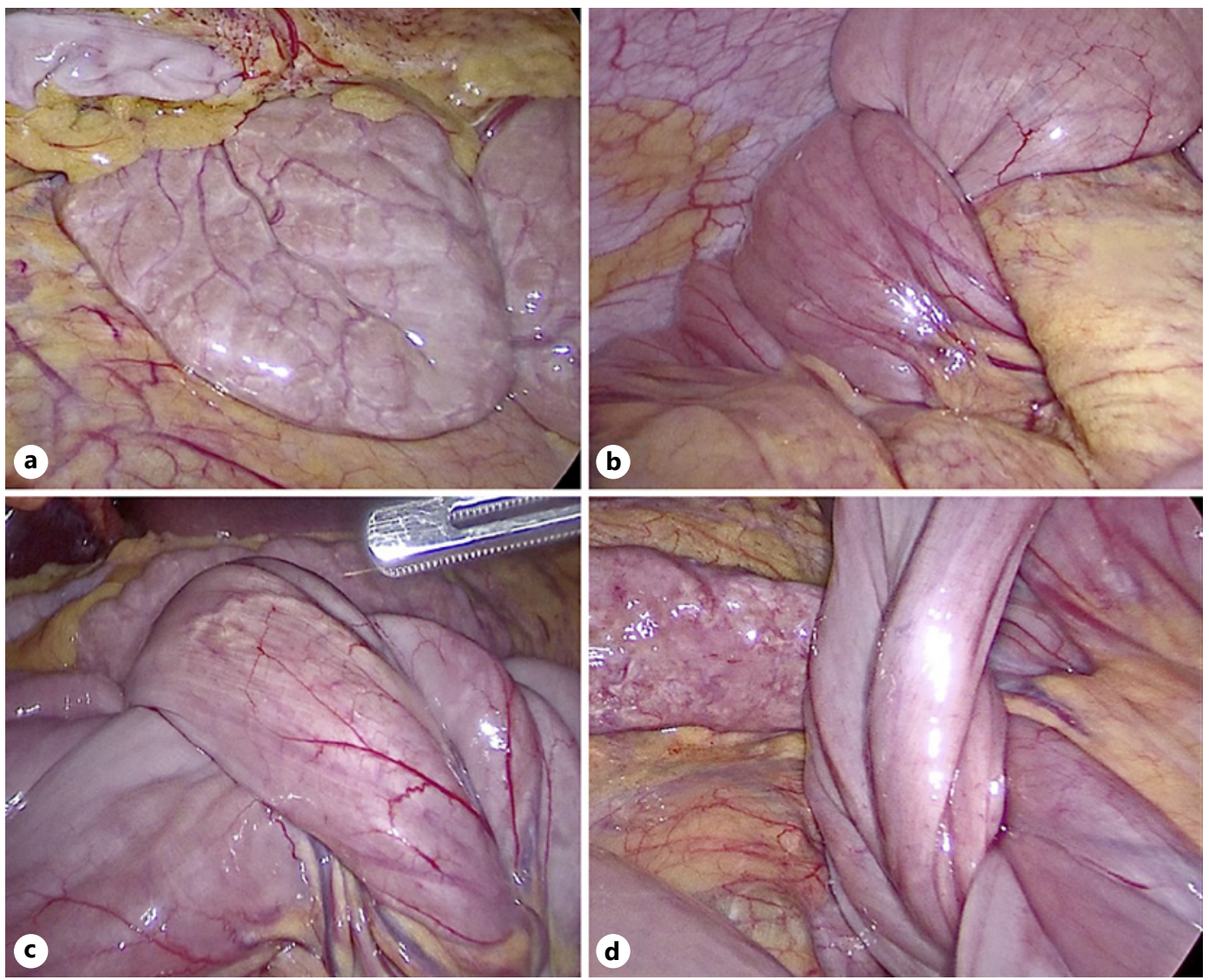

Fig. 2. a Small bowel appearance. b, c Jejunal invagination areas. $\mathbf{d}$ Attempt to correct the intestinal obstruction.

Another controversial aspect is the surgical approach. Classically, laparotomy was the most frequent approach because these patients were diagnosed with complete intestinal obstruction. However, in our case, we made an elective laparoscopic surgery since the patient had an incomplete obstruction. Another advantage of laparoscopy is the possibility to have an overall view of the bowel and ensure the absence of other lesions, as in this patient. To supplement that action, a mini laparotomy allows manual exploration of the entire bowel.

PJS prognosis is diverse depending on the diagnosis age, clinical symptoms, severe complications, surgical necessity, and malignancy risk. Consequently, mortality is increased, with a life expectancy of less than 65 years [14, 15].

In conclusion, PJS is an uncommon cause of recurrent abdominal pain. Patients suffering from this disease must be periodically evaluated since serious complications could develop. The worst complication in the acute period is intestinal obstruction due to intussusception, requiring surgical evaluation. While cancer develops in the long term, both define prognosis of the disease. Elective laparoscopy approach is a minimally invasive way to treat this disease, preventing intestinal excisions and the development of short bowel syndrome.

\section{Acknowledgements}

We acknowledge the entire General Surgery and Abdominal Organs Transplantation Department of the 12th October University Hospital, especially Dr. López and Dr. Gómez. 

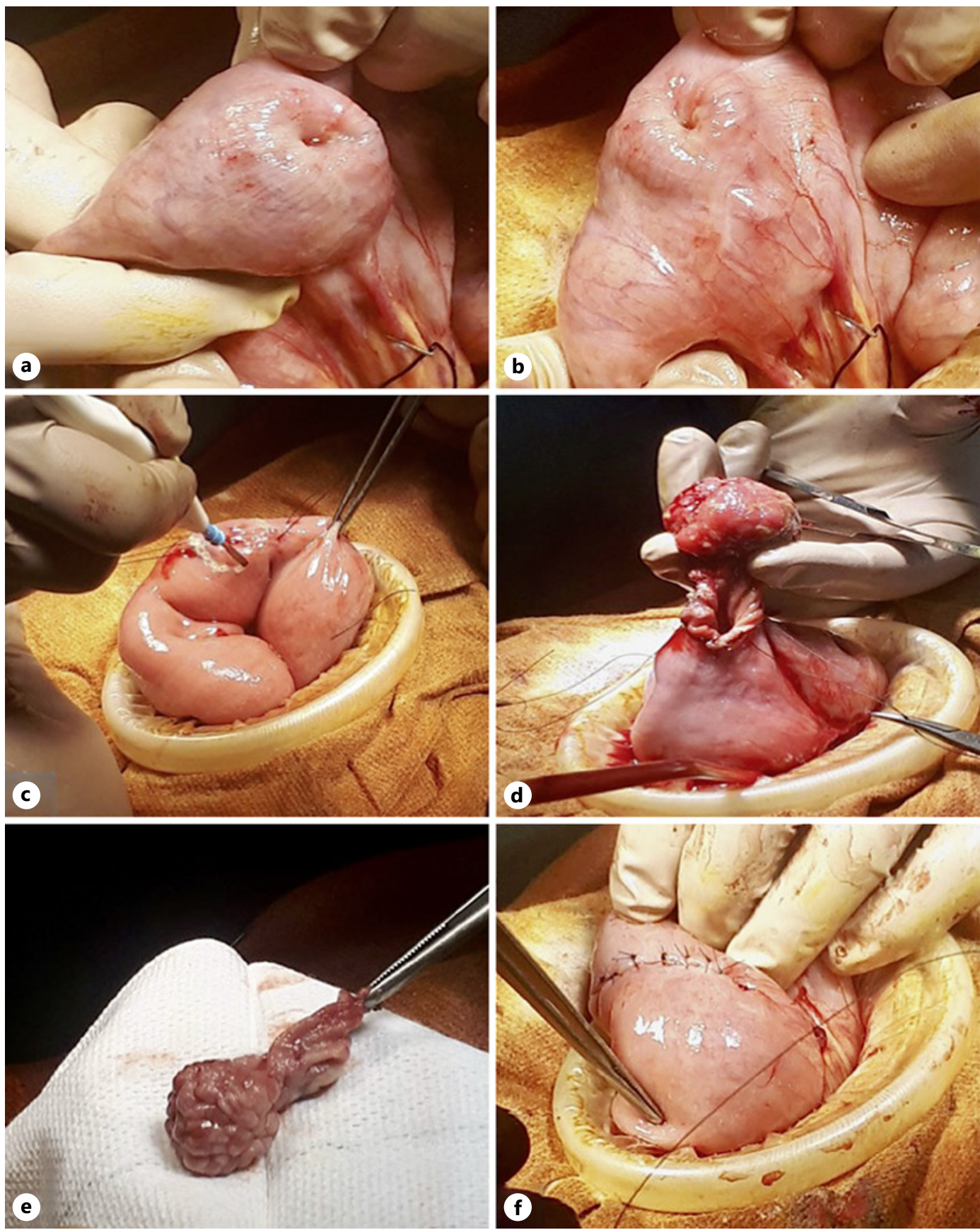

Fig. 3. a, b Intussusception areas of the small bowel marked with silk (Arrow). c Enterotomy was performed with the electric scalpel, surrounding the lesion. d, e Intestinal polyps in the intestinal lumen. $\mathbf{f}$ Enterotomies were transversely closed, with Endo-GIA staplers and absorbable 3/0 suture. Endo-GIA, endovascular gastrointestinal anastomosis.

\section{Statement of Ethics}

Written informed consent for publication of this case report including pictures was obtained from the patient. We have reported this case in compliance with the Declaration of Helsinki. The authors have no ethical conflicts to disclose. 


\section{Conflict of Interest Statement}

The authors have no conflicts of interest to declare.

\section{Funding Sources}

This study received no funding.

\section{Author Contributions}

A. García Picazo, B. López de la Torre, and A. Vivas are the main investigators in this case report and contributed to conceptualization, data collection, methodology, and writing of the manuscript. F.J. Garcia and E. Ferrero, read and revised the manuscript for publication. All the authors approved the final version of the manuscript.

\section{References}

1 Sanchez-Fortun C, Jover R. Poliposis colorrectales poco frecuentes, Síndrome de Peutz-Jeghers. Gh Continuada. 2010;9(2):55-9.

2 Cai HJ, Wang H, Cao N, Wang W, Sun XX, Huang B. Peutz-Jeghers syndrome with mesenteric fibromatosis: a case report and review of literature. World J Clin Cases. 2020;8(3):577-86.

3 Daniell J, Plazzer JP, Perera A, Macrae F. An exploration of genotype-phenotype link between Peutz-Jeghers syndrome and STK11: a review. Fam Cancer. 2018;17:421-7.

4 Giardiello FM, Brensinger JD, Tersmette AC, Goodman SN, Petersen GM, Booker SV, et al. Very high risk of cancer in familial Peutz-Jeghers syndrome. Gastroenterology. 2000;119:1447-53.

5 Kılıç S, Atıcı A, Soyköse-Açıkalın 0. Peutz-Jeghers syndrome: an unusual cause of recurrent intussusception in a 7-year-old boy. Turk J Pediatr. 2016;58:535-7.

6 Jeghers H, Mckusick VA, Katz KH. Generalized intestinal polyposis and melanin spots of the oral mucosa, lips and digits; a syndrome of diagnostic significance. N Engl J Med. 1949;241:993.

7 Meserve EE, Nucci MR. Peutz-jeghers syndrome: pathobiology, pathologic manifestations, and suggestions for recommending genetic testing in pathology reports. Surg Pathol Clin. 2016;9(2):243-68.

8 George SH, Milea A, Sowamber R, Chehade R, Tone A, Shaw PA. Loss of LKB1 and p53 synergizes to alter fallopian tube epithelial phenotype and high-grade serous tumorigenesis. Oncogene. 2016;35:59-68.

9 Hou L, Liu T, Wang J. Isoflurane suppresses the self-renewal of normal mouse neural stem cells in a p53-dependent manner by activating the Lkb1-p53-p21 signalling pathway. Mol Med Rep. 2015;12:7412-8.

10 Saito Y, Hinoi T, Ueno H, Kobayashi H, Konishi T, Ishida F, et al. Risk factors for the development of desmoid tumor after colectomy in patients with familial adenomatous polyposis: multicenter retrospective cohort study in Japan. Ann Surg Oncol. 2016;23:559-65.

11 Li BR, Sun T, Li J, Zhang YS, Ning SB, Jin XW, et al. Primary experience of small bowel polypectomy with balloonassisted enteroscopy in young pediatric Peutz-Jeghers syndrome patients. Eur J Pediatr. 2020;179(4):611.

12 Ugalde-Resano R, Bercholc-Urinowsky IJ, Escalona-Ramírez LE, Blanco-Torres JM, Paz-Muñoz MA, EscamillaRoque R, et al. Conservative surgical treatment in a patient with jejunal-jejunal intussusception secondary to Peutz-Jeghers syndrome. Case report. Acta Pediatr Mex. 2018;39(3):244-9.

13 Moluin N, Pedraza N, Padin J, Niveyro S, Tuhay G, Rumbo C, et al. Case report: spleen-preserving multivisceral transplant for Peutz-Jeghers syndrome. Transplant Proc. 2016;48(2):546-8.

14 Iwamuro M, Aoyama Y, Suzuki S, Kobayashi S, Toyokawa T, Moritou Y, et al. Long-term outcome in patients with a solitary Peutz-Jeghers polyp. Gastroenterol Res Pract. 2019;2019:8159072.

15 Mărginean CO, Melit LE, Patraulea F, Lunius S, Mărginean MO. Early onset Peutz-Jeghers syndrome, the importance of appropriate diagnosis and follow-up. Medicine. 2019;98(27):1-5. 